\title{
Perfil lipídico na adolescência: efeito de exposições intra-uterinas
}

\author{
Lipid profile in adolescents: effect of \\ intrauterine exposures
}

\section{Universidade Federal de Pelotas, Pelotas, Brasil. \\ Correspondência M. C. Restrepo Universidade Federal de Pelotas. \\ Rua Marechal Deodoro 1160 Pelotas, RS \\ 96020-220, Brasil. \\ klares7@gmail.com}

\begin{abstract}
This study assessed the effects of intrauterine growth restriction and risk factors for intrauterine growth restriction on blood lipids in adolescents from the 1982 birth cohort in Pelotas, Rio Grande do Sul State, Brazil. All male subjects were identified in 2000 when enrolling in the national army; $79 \%(n=2,250)$ were interviewed, and 2,089 provided blood samples. The following outcome variables were studied: total cholesterol and fractions (VLDL, $L D L$, and $H D L$ ), non- $H D L$ cholesterol, $L D L / H D L$ ratio, and serum triglycerides. The explanatory variables were intrauterine growth restriction, maternal pre-gestational body mass index (BMI), and maternal smoking during pregnancy. After adjusting for confounding variables, total, LDL, and non-HDL cholesterol levels were slightly and significantly higher among adolescents whose mothers were in the 3rd and 4th quartiles of pre-gestational BMI. However, these associations disappeared after adjusting for the adolescent's diet, schooling, and BMI. Other associations were not significant ( $p>0.05)$.
\end{abstract}

Fetal Growth Retardation; Cholesterol; Adolescent
María Clara Restrepo 1

Bernardo Lessa Horta 1

Denise Petrucci Gigante 1

\section{Introdução}

As doenças cardiovasculares são apontadas como a principal causa de óbito no mundo, sendo sua prevenção e controle um dos maiores desafios atuais para a saúde pública 1. Os níveis elevados de colesterol e triglicerídeos plasmáticos têm sido amplamente documentados como importantes fatores de risco modificáveis para essas doenças ${ }^{2}$.

Estudos epidemiológicos sugerem que o nível de lipídios plasmáticos pode ser determinado por exposições ocorridas na gestação ou nos primeiros anos de vida, mas as evidências ainda são controversas 3,4,5. As teorias de programação biológica e da origem precoce das doenças no adulto postulam que um ambiente fetal adverso (especialmente a subnutrição), durante um período crítico ou sensível do desenvolvimento, conduz a mudanças adaptativas na estrutura, metabolismo e fisiologia do feto 6 . Estudos em animais demonstraram que a carência nutricional no útero gera modificações na expressão de genes hepáticos de enzimas-chave (carnitina-palmitil-transferasa; acetil-CoA carboxilasa e proteína tri-funcional de $\beta$ oxidação) no metabolismo dos ácidos graxos. Tais mudanças ocorrem associadas com um aumento nos níveis de malonil-CoA e triglicerídeos séricos, conduzindo a um perfil lipídico alterado 7,8,9.

Em relação ao efeito do peso ao nascer (como marcador de crescimento fetal) sobre o metabo- 
lismo lipídico, Lawlor et al. 4, numa metanálise, observaram uma diminuição de 0,04mmol/L (IC95\%: -0,07; -0,02) no colesterol total para cada aumento de $1 \mathrm{~kg}$ no peso ao nascer no sexo masculino. Já no sexo feminino, foi estimada uma diminuição de 0,01 mmol/L (IC95\%: -0,04; 0,02). Ambos os resultados foram obtidos após ajuste para idade 4 . Contudo, cabe notar que esses estudos podem ter subestimado o efeito do crescimento intra-uterino, uma vez que o peso ao nascer é determinado por duas condições distintas, quais sejam, a duração da gestação e o crescimento fetal 10 .

São escassos os estudos realizados em países de média e baixa renda que avaliaram o efeito em longo prazo das condições intra-uterinas sobre o metabolismo lipídico. Nos países de média e baixa renda, além da prevalência de subnutrição ser mais elevada, espera-se que, entre as mães com baixo peso, a proporção de déficit nutricional e o retardo de crescimento intra-uterino sejam maiores. Nesse cenário de maior iniqüidade sócio-econômica, o risco atribuível de dislipidemia decorrente da subnutrição (de acordo com a nossa hipótese) é maior do que em países de alta renda.

O presente estudo teve como objetivo avaliar o efeito do retardo de crescimento intra-uterino e de alguns fatores de risco desse retardo (índice de massa corporal materno pré-gestacional e tabagismo materno) sobre o perfil lipídico de adolescentes da coorte de 1982 da cidade de Pelotas, Rio Grande do Sul, Brasil.

\section{Métodos}

A presente pesquisa está vinculada ao estudo de coorte das crianças nascidas em 1982, em Pelotas, cidade de porte médio localizada no extremo Sul do Brasil. A população é na sua maioria branca, descendente do sul da Europa. Esta é uma parte do Brasil relativamente desenvolvida, mas com amplas desigualdades sócio-econômicas. Segundo estimativas do Censo Demográfico (http:// www.ibge.gov.br/home/estatistica/populacao/ contagem2007, acessado em 01/Out/2008), a população urbana aumentou de cerca de $210 \mathrm{mil}$, em 1982, para aproximadamente 340 mil habitantes, em 2007.

Entre 1ㅇ de janeiro e 31 de dezembro de 1982, todos os 6.011 nascimentos hospitalares da cidade foram identificados (aproximadamente 99\% de todos os nascimentos) e as 5.914 crianças nascidas vivas, cujas famílias residiam na área urbana, foram incluídas no estudo. Um questionário padronizado foi aplicado à mãe por ocasião do nascimento, visando obter informações quanto a variáveis demográficas, sócio-econômicas e de assistência à gestação e ao parto. Os recém-nascidos foram pesados com balanças pediátricas (Filizola S.A. Pesagem e Automação, São Paulo, Brasil), suas mães tiveram seu peso e altura aferidos 11. A população em estudo foi acompanhada por inúmeras vezes. Maiores detalhes das metodologias do estudo perinatal e dos acompanhamentos estão disponíveis em outras publicações 11,12.

Entre janeiro e abril de 2000, todos os homens nascidos em 1982 deveriam comparecer à junta de alistamento militar, oportunidade em que um entrevistador coletou informações que permitiram a identificação do adolescente como um membro da coorte. No momento do exame médico do alistamento militar (de julho a setembro de 2000), os adolescentes foram convidados a responder a um questionário e a doar uma amostra de sangue. Os recrutas compareceram ao quartel às $6 \mathrm{~h}$ da manhã e os exames foram realizados. As amostras de sangue foram coletadas por venopunção entre as 10h30min e o meio-dia, após a realização dos exames médicos, intelectuais e antropométricos. Dessa forma, estima-se que os membros da coorte estavam com, pelo menos, cinco a sete horas de jejum 5,11.

As seguintes variáveis dependentes foram estudadas: colesterol total e suas frações (VLDL, LDL e HDL); colesterol não HDL, razão LDL/HDL e triglicerídeos plasmáticos. O colesterol total, HDL e triglicerídeos foram medidos usando métodos enzimáticos (Dimension Clinical Chemestry system; Dade Behring). O colesterol VLDL foi estimado com base nos níveis de triglicerídeos. $\mathrm{O}$ colesterol LDL foi estimado pela fórmula de Friedewald et al 13 [colesterol total - (HDL + Triglicerídeos/5)]. O colesterol não-HDL foi calculado pela diferença entre o colesterol total e o HDL 2. Todos os valores foram expressos em $\mathrm{mg} / \mathrm{dL}$, exceto para razão LDL/HDL.

As variáveis independentes estudadas foram: retardo de crescimento intra-uterino, índice de massa corporal (IMC) materno pré-gestacional e tabagismo materno na gravidez. O retardo de crescimento intra-uterino foi definido como o peso ao nascer de acordo com idade gestacional e sexo abaixo do percentil 10 da curva de Willia$\mathrm{ms}$ et al. 14. Todas as variáveis foram coletadas no estudo perinatal, em 1982. O IMC materno pré-gestacional foi calculado mediante altura (medida pela equipe de pesquisa) e peso materno no início da gestação (coletado na carteira de pré-natal ou referido pelas mães). O tabagismo materno foi coletado em categorias relacionadas à freqüência e, para este estudo, estipularam-se três categorias, a saber: não fumou, fumou parte da gravidez, fumou toda a gravidez. O tabagismo 
também foi classificado de acordo com a quantidade de cigarros fumados num dia. Assim, os participantes do estudo puderam ser classificados da seguinte maneira em relação à intensidade quanto ao fumo: não fumou, fumou de 1-14 cigarros/dia, fumou 15 ou mais cigarros/dia.

As seguintes variáveis foram consideradas como possíveis fatores de confusão: idade da mãe no momento do nascimento da criança (categorizada em $<20,20-29$ e $\geq 30$ ), cor da pele materna auto-referida (categorizado em branca e não branca), escolaridade materna (categorizada em 0-4, 5-8, 9-11 e $\geq 12$ anos de estudo completos), renda familiar ao nascer (classificada como $\leq 1,1,1-3,0$ e $>3,0$ salários mínimos) e paridade (categorizada em 0, 1, 2, 3 ou mais filhos). Todas essas variáveis foram referidas pela mãe durante o estudo perinatal. A renda familiar ao nascer (classificada como $\leq 3$ salários mínimos e $>3$ salários mínimos) foi avaliada como possível modificador de efeito. Já que não houve evidência de interação $(\leq 20 \%)$ pela renda familiar, as análises não foram apresentadas estratificadas por esta variável.

Para a análise bruta dos dados, foram utilizados o teste $\mathrm{t}$ e a análise de variância. Na análise multivariável, foi utilizada a regressão linear múltipla. Em todas as análises foi utilizado um nível de significância de 5\%. Foram considerados como possíveis fatores de confusão aquelas variáveis que estavam associadas com o desfecho num nível de significância menor ou igual a $20 \%$. As análises foram realizadas utilizando o programa Stata 9.0 (Stata Corp., College Station, Estados Unidos).

Considerando que os dados utilizados neste estudo já estavam coletados, o cálculo do poder estatístico foi realizado a posteriori. O banco de dados incluiu aproximadamente 2.083 adolescentes, o que indica que, com o tamanho da amostra, o estudo teve um poder estatístico de $80 \%$ para detectar como estatisticamente significativa uma diferença de pelo menos $7 \mathrm{mg} / \mathrm{dL}$ entre os grupos expostos (adolescentes cujas mães tiveram IMC pré-gestacional $<20 \mathrm{~kg} / \mathrm{m}^{2}$; adolescentes cujas mães fumaram na gravidez e adolescentes com retardo de crescimento intrauterino ao nascer) e não expostos.

Sob o ponto de vista ético, garantiu-se a confidencialidade de todas as informações coletadas e se obteve o consentimento dos responsáveis ou do indivíduo em todas as fases do estudo (verbal em 1982 e escrito em 2000). O protocolo do estudo foi aprovado pelo Comitê de Ética Médica da Universidade Federal de Pelotas.

\section{Resultados}

Em 2000, foram entrevistados 2.250 dos 3.037 indivíduos do sexo masculino membros da coorte original. Somados aos 143 indivíduos do sexo masculino que faleceram até esse ano, foi obtida uma taxa de acompanhamento de $79 \%$. As perdas foram mais freqüentes entre adolescentes nascidos nas famílias mais pobres (27\%) e mais ricas (22\%), quando comparados com adolescentes nascidos nas famílias das classes intermediárias. Igualmente, observaram-se perdas maiores entre adolescentes cujas mães tinham uma ou mais das seguintes características: idade inferior a 20 anos (24\%); de 0-4 ou de 5-9 anos de estudo; IMC pré-gestacional inferior a $22 \mathrm{~kg} / \mathrm{m}^{2}(23 \%)$; fumo durante toda a gravidez (24\%) 11 .

Dos 2.250 indivíduos entrevistados, 2.083 forneceram uma amostra de sangue para a medida do colesterol total. Em 24 indivíduos não se obteve uma quantidade suficiente para medir os outros componentes do perfil lipídico. A Tabela 1 apresenta a média e o desvio-padrão dos lipídios plasmáticos estudados. A Tabela 2 apresenta o colesterol total, HDL e não-HDL de acordo com características demográficas, sócio-econômicas, antropométricas e comportamentais da amostra. Verificou-se que a média de colesterol total e de colesterol não-HDL foi maior entre adolescentes cujas mães tinham maior escolaridade e maior renda familiar, e menor entre adolescentes cujas mães tinham menor IMC materno pré-gestacional. A média de colesterol HDL foi menor entre os adolescentes de mães brancas.

A Tabela 3 mostra as análises bruta e ajustada para colesterol total, colesterol HDL e triglicerídeos. Em média, o colesterol total foi maior entre os adolescentes cujas mães estavam no 3 o

Tabela 1

Distribuição da amostra conforme níveis de lipídios plasmáticos aos 18 anos em homens pertencentes ao estudo de coorte de nascimento em Pelotas, Rio Grande do Sul, Brasil, 1982.

\begin{tabular}{lccc}
\hline Variável & $\mathbf{n}$ & Média & DP \\
\hline Colesterol total & 2.083 & 143,1 & 29,0 \\
Colesterol VLDL & 2.059 & 14,8 & 9,6 \\
Colesterol LDL & 2.059 & 88,2 & 25,1 \\
Colesterol HDL & 2.059 & 40,1 & 9,7 \\
Colesterol não-HDL & 2.059 & 103,0 & 27,3 \\
Razão LDL/HDL & 2.059 & 2,3 & 0,8 \\
Triglicerídeos & 2.059 & 76,1 & 48,0 \\
\hline
\end{tabular}

DP: desvio-padrão.

Nota: todos os valores são expressos em mg/dL, exceto para razão LDL/HDL. 
Tabela 2

Distribuição do colesterol total, HDL e não-HDL em adolescentes do sexo masculino conforme características da amostra. Coorte de nascimento de 1982, Pelotas, Rio Grande do Sul, Brasil.

\begin{tabular}{|c|c|c|c|c|c|c|}
\hline \multirow[t]{2}{*}{ Variável } & \multicolumn{2}{|c|}{ Colesterol total } & \multicolumn{2}{|c|}{ HDL } & \multicolumn{2}{|c|}{ Não-HDL } \\
\hline & $\mathbf{n}$ & Média (DP) & n & Média (DP) & $\mathbf{n}$ & Média (DP) \\
\hline \multicolumn{7}{|l|}{ Cor da pele da mãe } \\
\hline Branca & 1.720 & $143,1(29,3)$ & 1.706 & $39,6(9,5)$ & 1.706 & $103,5(27,7)$ \\
\hline Parda/Preta & 361 & $143,0(27,9)$ & 351 & $42,2(10,0)$ & 351 & $101,0(25,2)$ \\
\hline Valor de $\mathrm{p}$ & & 0,96 * & & 0,001 * & & $0,2 * \star$ \\
\hline \multicolumn{7}{|l|}{ Idade mãe (anos) } \\
\hline$<20$ & 301 & $140,2(26,7)$ & 295 & $39,3(9,4)$ & 295 & $100,9(25,2)$ \\
\hline $20-29$ & 1.215 & $143,0(29,2)$ & 1.202 & $40,2(9,7)$ & 1.202 & $102,8(27,2)$ \\
\hline$\geq 30$ & 567 & $144,8(29,8)$ & 562 & $40,1(9,8)$ & 562 & $104,6(28,6)$ \\
\hline Valor de p & & 0,08 * & & 0,4 * & & 0,2 * \\
\hline \multicolumn{7}{|c|}{ Escolaridade materna (anos completos) } \\
\hline $0-4$ & 655 & $139,1(27,7)$ & 646 & $39,6(10,0)$ & 646 & $99,5(25,5)$ \\
\hline $5-8$ & 920 & $142,9(28,1)$ & 910 & $40,5(9,7)$ & 910 & $102,3(26,3)$ \\
\hline $9-11$ & 225 & $145,0(31,8)$ & 222 & $39,2(9,5)$ & 222 & $105,7(30,5)$ \\
\hline$\geq 12$ & 279 & $151,6(30,9)$ & 277 & $40,4(8,8)$ & 277 & $111,3(30,5)$ \\
\hline Valor de $\mathrm{p}$ & & 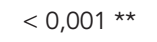 & & 0,2 * & & $<0,001$ ** \\
\hline \multicolumn{7}{|c|}{ Renda familiar (salários mínimos) } \\
\hline$<1,0$ & 389 & $140,9(27,4)$ & 381 & $40,4(9,6)$ & 381 & $100,4(24,9)$ \\
\hline $1,1-3,0$ & 1.034 & $140,8(28,6)$ & 1.021 & $39,6(9,8)$ & 1.021 & $101,2(26,5)$ \\
\hline $3,0-6,0$ & 414 & $144,7(27,8)$ & 411 & $40,3(9,3)$ & 411 & $104,4(27,2)$ \\
\hline$>6,0$ & 238 & $153,8(32,8)$ & 238 & $41,0(9,7)$ & 238 & $112,8(32,3)$ \\
\hline Valor de $\mathrm{p}$ & & $<0,001 \star \star$ & & 0,2 * & & 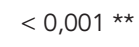 \\
\hline \multicolumn{7}{|l|}{ Paridade } \\
\hline Nenhum & 841 & $144,7(29,1)$ & 830 & $40,1(9,3)$ & 830 & $104,7(27,1)$ \\
\hline 1 & 592 & $142,9(29,2)$ & 581 & $40,2(9,9)$ & 581 & $103,0(27,6)$ \\
\hline 2 & 345 & $141,4(27,3)$ & 344 & $40,3(9,8)$ & 344 & $101,0(26,5)$ \\
\hline 3 ou mais & 304 & $140,7(30,0)$ & 303 & $39,6(10,0)$ & 303 & $100,9(27,8)$ \\
\hline Valor de $\mathrm{p}$ & & 0,1 * & & 0,8 * & & 0,07 * \\
\hline \multicolumn{7}{|l|}{ IMC materno pré-gestacional } \\
\hline$\leq 20,1$ & 436 & $142,7(27,1)$ & 432 & $39,9(9,3)$ & 432 & $102,8(24,5)$ \\
\hline $20,2-21,9$ & 441 & $140,2(26,9)$ & 436 & $39,7(9,3)$ & 436 & $100,4(25,5)$ \\
\hline $22,0-24,6$ & 460 & $145,9(30,5)$ & 453 & $40,7(9,5)$ & 453 & $105,4(29,3)$ \\
\hline$\geq 24,7$ & 460 & $144,6(30,3)$ & 453 & $39,8(9,8)$ & 453 & $104,6(28,4)$ \\
\hline Valor de $\mathrm{p}$ & & $0,02 * \star$ & & 0,4 * & & $0,04 * *$ \\
\hline \multicolumn{7}{|l|}{ Tabagismo materno } \\
\hline Não fumou & 1.369 & $143,4(29,7)$ & 1.348 & $40,3(9,8)$ & 1.348 & $103,3(28,0)$ \\
\hline Fumou parte da gravidez & 170 & $143,9(30,2)$ & 169 & $40,2(9,3)$ & 169 & $103,7(27,9)$ \\
\hline Fumou toda a gravidez & 538 & $141,9(26,8)$ & 536 & $39,5(9,3)$ & 536 & $102,3(25,4)$ \\
\hline Valor de $\mathrm{p}$ & & $0,5 * \star$ & & 0,3 * & & $0,8 * \star$ \\
\hline \multicolumn{7}{|c|}{ Retardo de crescimento intra-uterino $* \star \star$} \\
\hline Não & 1.539 & $143,2(29,0)$ & 1.526 & $40,0(9,4)$ & 1.526 & $103,2(27,5)$ \\
\hline $\operatorname{Sim}$ & 143 & $141,5(28,9)$ & 143 & $38,9(10,3)$ & 143 & $102,6(26,9)$ \\
\hline Valor de $\mathrm{p}$ & & 0,5 * & & 0,2 * & & 0,8 * \\
\hline
\end{tabular}

IMC: índice de massa corporal.

* Teste de ANOVA para variâncias homogêneas;

** Teste de Kruskal wallis para variâncias heterogêneas;

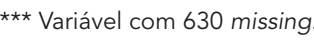


Análise bruta e ajustada da associação entre variáveis pré-natais e neonatais com colesterol total, HDL e triglicerídeos aos 18 anos em adolescentes do sexo masculino pertencentes ao estudo de coorte de nascimento em Pelotas, Rio Grande do Sul, Brasil, 1982.

\begin{tabular}{|c|c|c|c|c|c|c|c|c|c|}
\hline \multirow[t]{3}{*}{ Variável } & \multicolumn{3}{|c|}{ Colesterol total (mg/dL) } & \multicolumn{3}{|c|}{ Colesterol HDL (mg/dL) } & \multicolumn{3}{|c|}{ Triglicerídeos (mg/dL) } \\
\hline & $\mathbf{n}$ & Bruta & Ajustada & $\mathbf{n}$ & Bruta & Ajustada & $\mathbf{n}$ & Bruta & Ajustada \\
\hline & & $\beta$ (IC95\%) & $\beta$ (IC95\%) & & $\beta$ (IC95\%) & $\beta$ (IC95\%) & & $\beta$ (IC95\%) & $\beta$ (IC95\%) \\
\hline \multicolumn{10}{|l|}{ IMC materno pré-gestacional * } \\
\hline$\leq 20,1$ & 436 & $0,0(-)$ & $0,0(-)$ & 432 & $0,0(-)$ & $0,0(-)$ & 432 & $0,0(-)$ & $0,0(-)$ \\
\hline $20,2-21,9$ & 441 & $-2,5(-6,3 ; 1,3)$ & $-2,2(-6,0 ; 1,6)$ & 436 & $-0,1(-1,4 ; 1,1)$ & $-0,1(-1,3 ; 1,2)$ & 436 & $2,9(-3,4 ; 9,2)$ & $3,3(-2,9 ; 9,6)$ \\
\hline $22,0-24,6$ & 460 & $3,2(-0,6 ; 7,0)$ & $3,5(-0,2 ; 7,3)$ & 453 & $0,9(-0,4 ; 2,1)$ & $0,8(-0,4 ; 2,1)$ & 453 & $8,4(2,2 ; 14,7)$ & $8,3(2,2 ; 14,5)$ \\
\hline$\geq 24,7$ & 460 & $1,9(-1,9 ; 5,7)$ & $2,5(-1,4 ; 6,5)$ & 453 & $-0,1(-1,3 ; 1,2)$ & $-0,2(-1,4 ; 1,1)$ & 453 & $3,4(-2,9 ; 9,6)$ & $4,5(-1,7 ; 10,7)$ \\
\hline Valor de p & & 0,02 & 0,01 & & 0,4 & 0,4 & & 0,06 & 0,07 \\
\hline \multicolumn{10}{|l|}{ Tabagismo materno ** } \\
\hline Não fumou & 1.369 & $0,0(-)$ & $0,0(-)$ & 1.348 & $0,0(-)$ & $0,0(-)$ & 1.348 & $0,0(-)$ & $0,0(-)$ \\
\hline Fumou parte da gravidez & 170 & $0,5(-4,1 ; 5,2)$ & $1,4(-3,5 ; 6,4)$ & 169 & $-0,1(-1,6 ; 1,5)$ & $-0,1(-1,6 ; 1,5)$ & 169 & $0,4(-7,3 ; 8,0)$ & $4,2(-3,9 ; 12,3)$ \\
\hline Fumou toda a gravidez & 538 & $1,51(-4,4 ; 1,4)$ & $0,4(-2,8 ; 3,6)$ & 536 & $-0,7(-1,6 ; 0,3)$ & $-0,7(-1,7 ; 0,3)$ & 536 & $-2,6(-7,4 ; 2,2)$ & $0,7(-4,5 ; 5,8)$ \\
\hline Valor de $\mathrm{p}$ & & $0,3 * \star \star$ & $0,8 * \star \star$ & & 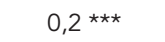 & $0,2 * \star \star$ & & $0,3 * \star \star$ & $0,8 * \star \star$ \\
\hline \multicolumn{10}{|l|}{ Retardo de crescimento } \\
\hline \multicolumn{10}{|l|}{ intra-uterino \# } \\
\hline Não & 1.539 & $0,0(-)$ & $0,0(-)$ & 1.526 & $0,0(-)$ & $0,0(-)$ & 1.526 & $0,0(-)$ & $0,0(-)$ \\
\hline Sim & 143 & $-1,7(-6,7 ; 3,3)$ & $0,2(-5,2 ; 5,5)$ & 143 & $-1,1(-2,7 ; 0,5)$ & $-1,1(-2,8 ; 0,5)$ & 143 & $-0,7(-9,0 ; 7,6)$ & $3,3(-5,3 ; 11,9)$ \\
\hline Valor de $p$ & & 0,5 & 1,0 & & 0,2 & 0,2 & & 0,9 & 0,4 \\
\hline
\end{tabular}

IC95\%: intervalo de 95\% de confiança; IMC: índice de massa corporal.

* Ajustada para cor da pele da mãe, renda familiar ao nascer, escolaridade materna, paridade, tabagismo materno;

** Ajustada para cor da pele da mãe, renda familiar ao nascer, escolaridade materna, paridade, IMC materno pré-gestacional;

*** Teste Wald de tendência linear;

\# Ajustada para cor da pele da mãe, renda familiar ao nascer, escolaridade materna, paridade, IMC materno pré-gestacional, tabagismo materno.

Nota: todos os valores são expressos em mg/dL.

e 4o quartil de IMC pré-gestacional; no entanto, não foi observada uma relação linear. Na análise bruta e ajustada, não houve diferenças significativas nos níveis de colesterol HDL e triglicerídeos entre as categorias de IMC materno prégestacional. O tabagismo materno na gestação (conforme freqüência e intensidade) e o retardo de crescimento intra-uterino não apresentaram associação com o colesterol total, colesterol HDL e triglicerídeos.

A Tabela 4 apresenta as análises bruta e ajustada para colesterol VLDL, colesterol LDL, colesterol não-HDL e razão LDL/HDL. Similarmente ao que foi constatado para o colesterol total, o colesterol LDL foi em média maior nos adolescentes cujas mães pertenciam ao 3o e 4o quartil de IMC pré-gestacional $(p=0,05)$. Entretanto, não foi observada tendência linear $(\mathrm{p}$ de tendência $=$ $0,1)$ de aumento do colesterol LDL com o aumento do IMC materno. Uma associação similar foi observada entre o colesterol não-HDL e o IMC materno pré-gestacional. Da mesma forma como verificado na Tabela 3, o tabagismo materno (conforme freqüência e intensidade) e o retardo de crescimento intra-uterino não estiveram associados com os níveis de colesterol VLDL, LDL, não-HDL e razão LDL/HDL.

\section{Discussão}

No presente estudo, avaliamos o efeito da programação do crescimento intra-uterino sobre o metabolismo lipídico em adolescentes aos 18 anos de idade. Além de avaliar o efeito do peso ao nascer de acordo com a idade gestacional, variáveis reconhecidamente associadas à restrição do crescimento intra-uterino 15,16 foram incluídas na análise, mas não encontramos qualquer efeito de programação em longo prazo, como proposto pela teoria de Barker.

O desenho prospectivo deste estudo torna-o menos suscetível a um viés de informação, pois as exposições (peso ao nascer para idade gestacional, IMC materno pré-gestacional e tabagismo materno durante a gestação) foram medidas no momento do nascimento e, dessa forma, não é possível creditar a ausência de associações sig- 
Análise bruta e ajustada da associação entre exposições precoces e colesterol VLDL, LDL, não-HDL e razão LDL/HDL em adolescentes do sexo masculino pertencentes ao estudo de coorte de nascimento em Pelotas, Rio Grande do Sul, Brasil, 1982.

\begin{tabular}{|c|c|c|c|c|c|c|c|c|c|c|c|c|}
\hline \multirow[t]{2}{*}{ Variável } & \multicolumn{3}{|c|}{ Colesterol VLDL } & \multicolumn{3}{|c|}{ Colesterol LDL } & \multicolumn{3}{|c|}{ Colesterol não-HDL } & \multicolumn{3}{|c|}{ Razão LDL/HDL } \\
\hline & $\mathrm{n}$ & $\begin{array}{c}\text { Bruta } \\
\beta(\text { IC95\%) }\end{array}$ & $\begin{array}{l}\text { Ajustada } \\
\beta \text { (IC95\%) }\end{array}$ & $\mathrm{n}$ & $\begin{array}{c}\text { Bruta } \\
\beta \text { (IC95\%) }\end{array}$ & $\begin{array}{l}\text { Ajustada } \\
\beta \text { (IC95\%) }\end{array}$ & $n$ & $\begin{array}{c}\text { Bruta } \\
\beta(\text { IC95\%) }\end{array}$ & $\begin{array}{l}\text { Ajustada } \\
\beta \text { (IC95\%) }\end{array}$ & $\mathrm{n}$ & $\begin{array}{c}\text { Bruta } \\
\beta \text { (IC95\%) }\end{array}$ & $\begin{array}{c}\text { Ajustada } \\
\beta \text { (IC95\%) }\end{array}$ \\
\hline \multicolumn{13}{|l|}{$\begin{array}{l}\text { IMC materno } \\
\text { pré-gestacional * }\end{array}$} \\
\hline$\leq 20,1$ & 432 & $0,0(-)$ & $0,0(-)$ & 432 & $0,0(-)$ & $0,0(-)$ & 432 & $0,0(-)$ & $0,0(-)$ & 432 & $0,0(-)$ & $0,0(-)$ \\
\hline $20,2-21,9$ & 436 & $\begin{array}{c}0,6 \\
(-0,7 ; 1,8)\end{array}$ & $\begin{array}{c}0,7 \\
(-0,6 ; 1,9)\end{array}$ & 436 & $\begin{array}{c}-3,0 \\
(-6,3 ; 0,3)\end{array}$ & $\begin{array}{c}-2,8 \\
(-6,1 ; 0,5)\end{array}$ & 436 & $\begin{array}{c}-2,4 \\
(-6,0 ; 1,2)\end{array}$ & $\begin{array}{c}-2,2 \\
(-5,8 ; 1,4)\end{array}$ & 436 & $\begin{array}{c}-0,1 \\
(-0,2 ; 0,0)\end{array}$ & $\begin{array}{c}-0,1 \\
(-0,2 ; 0,0)\end{array}$ \\
\hline $22,0-24,6$ & 453 & $\begin{array}{c}1,7 \\
(0,4 ; 2,9)\end{array}$ & $\begin{array}{c}1,7 \\
(0,4 ; 2,9)\end{array}$ & 453 & $\begin{array}{c}0,9 \\
(-2,4 ; 4,2)\end{array}$ & $\begin{array}{c}0,9 \\
(-2,3 ; 4,2)\end{array}$ & 453 & $\begin{array}{c}2,6 \\
(-1,0 ; 6,2)\end{array}$ & $\begin{array}{c}2,6 \\
(-0,9 ; 6,2)\end{array}$ & 453 & $\begin{array}{c}0,0 \\
(-0,1 ; 0,1)\end{array}$ & $\begin{array}{c}0,0 \\
(-0,1 ; 0,1)\end{array}$ \\
\hline$\geq 24,7$ & 453 & $\begin{array}{c}0,7 \\
(-0,6 ; 1,9)\end{array}$ & $\begin{array}{c}0,9 \\
(-0,3 ; 2,2)\end{array}$ & 453 & $\begin{array}{c}1,1 \\
(-2,2 ; 4,4)\end{array}$ & $\begin{array}{c}1,6 \\
(-1,7 ; 4,8)\end{array}$ & 453 & $\begin{array}{c}1,8 \\
(-1,8 ; 5,3)\end{array}$ & $\begin{array}{c}2,4 \\
(-1,2 ; 5,9)\end{array}$ & 453 & $\begin{array}{c}0,0 \\
(-0,1 ; 0,1)\end{array}$ & $\begin{array}{c}0,1 \\
(0,0 ; 0,2)\end{array}$ \\
\hline \multicolumn{13}{|l|}{$\begin{array}{l}\text { Tabagismo } \\
\text { materno ** }\end{array}$} \\
\hline Não fumou & 1.348 & $0,0(-)$ & $0,0(-)$ & 1.348 & $0,0(-)$ & $0,0(-)$ & 1.348 & $0,0(-)$ & $0,0(-)$ & 1348 & $0,0(-)$ & $0,0(-)$ \\
\hline $\begin{array}{l}\text { Fumou parte } \\
\text { da gravidez }\end{array}$ & 169 & $\begin{array}{c}0,1 \\
(-1,5 ; 1,6)\end{array}$ & $\begin{array}{c}0,8 \\
(-0,8 ; 2,5)\end{array}$ & 169 & $\begin{array}{c}0,4 \\
(-3,7 ; 4,4)\end{array}$ & $\begin{array}{c}0,5 \\
(-3,8 ; 4,8)\end{array}$ & 169 & $\begin{array}{c}0,4 \\
(-4,0 ; 4,8)\end{array}$ & $\begin{array}{c}1,4 \\
(-3,3 ; 6,0)\end{array}$ & 169 & $\begin{array}{c}0,0 \\
(-0,1 ; 0,1)\end{array}$ & $\begin{array}{c}0,0 \\
(-0,1 ; 0,2)\end{array}$ \\
\hline Toda a gravidez & 536 & $\begin{array}{c}0,53 \\
(-1,5 ; 0,4)\end{array}$ & $\begin{array}{c}0,1 \\
(-0,9 ; 1,1)\end{array}$ & 536 & $\begin{array}{c}0,44 \\
(-3,0 ; 2,1)\end{array}$ & $\begin{array}{c}0,1 \\
(-2,7 ; 2,8)\end{array}$ & 536 & $\begin{array}{c}-1,0 \\
(-3,7 ; 1,8)\end{array}$ & $\begin{array}{c}0,3 \\
(-2,7 ; 3,2)\end{array}$ & 536 & $\begin{array}{c}0,0 \\
(-0,1 ; 0,1)\end{array}$ & $\begin{array}{c}0,0 \\
(-0,1 ; 0,1)\end{array}$ \\
\hline Valor de $\mathrm{p}$ & & $0,3 * \star \star$ & 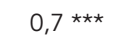 & & $0,8 * \star \star$ & $0,9 * \star \star$ & & $0,5^{\star \star \star}$ & $0,8 * \star \star$ & & $0,5 * \star \star$ & $0,5 * \star \star$ \\
\hline $\begin{array}{l}\text { Retardo de } \\
\text { crescimento } \\
\text { intra-uterino \# }\end{array}$ & & & & & & & & & & & & \\
\hline Não & 1.526 & $0,0(-)$ & $0,0(-)$ & 1.526 & $0,0(-)$ & $0,0(-)$ & 1.526 & $0,0(-)$ & $0,0(-)$ & 1.526 & $0,0(-)$ & $0,0(-)$ \\
\hline Sim & 143 & $\begin{array}{c}0,13 \\
(-1,8 ; 1,5)\end{array}$ & $\begin{array}{c}0,8 \\
(-1,0 ; 2,5)\end{array}$ & 143 & $\begin{array}{c}0,53 \\
(-4,8 ; 3,8)\end{array}$ & $\begin{array}{c}0,7 \\
(-4,0 ; 5,3)\end{array}$ & 143 & $\begin{array}{c}0,66 \\
(-5,4 ; 4,0)\end{array}$ & $\begin{array}{c}1,5 \\
(-3,5 ; 6,6)\end{array}$ & 143 & $\begin{array}{c}0,1 \\
(-0,1 ; 0,2)\end{array}$ & $\begin{array}{c}0,1 \\
(-0,1 ; 0,2)\end{array}$ \\
\hline Valor de $p$ & & 0,9 & 0,4 & & 0,8 & 0,8 & & 0,8 & 0,6 & & 0,4 & 0,3 \\
\hline
\end{tabular}

IC95\%: intervalo de 95\% de confiança; IMC: índice de massa corporal.

* Ajustada para cor da pele da mãe, renda familiar ao nascer, escolaridade materna, paridade, tabagismo materno;

** Ajustada para cor da pele da mãe, renda familiar ao nascer, escolaridade materna, paridade, IMC materno pré-gestacional;

*** Teste Wald de tendência linear;

\# Ajustada para cor da pele da mãe, renda familiar ao nascer, escolaridade materna, paridade, IMC materno pré-gestacional, tabagismo materno.

IMC materno pré-gestacional: $\leq 20,1$ (quartil inferior); 20,2-21,9 ( $2^{\circ}$ quartil); 22,0-24,6 ( $3^{\circ}$ quartil); $\geq 24,7$ (quartil superior).

Nota: todos os valores são expressos em $\mathrm{mg} / \mathrm{dL}$, exceto a razão LDL/HDL.

nificativas a um erro de classificação não diferencial. Além disso, as elevadas taxas de acompanhamento e a base populacional da coorte contribuíram para prevenir o viés de seleção.

Entre as principais limitações do nosso estudo está o fato de que as amostras não foram coletadas após um período de jejum de 12 horas. Uma vez que os jovens tinham de chegar às $6 \mathrm{~h}$ da manhã para o exame médico de seleção e a coleta de sangue seria feita entre as $10 \mathrm{~h} 30 \mathrm{~min}$ e o meio dia, o tempo mínimo de jejum foi de cinco horas. Nesse período, os níveis de triglicerídeos plasmáticos podem ter sido afetados pela alimentação prévia. Por conseguinte, não excluímos a pos- sível presença de um erro de classificação não diferencial no desfecho, que conduziria a uma subestimação do efeito das exposições sobre os níveis de triglicerídeos e, portanto, a uma possível ausência de associação. Por outro lado, não se encontrou uma tendência linear nos efeitos estimados, fato que reforça a possibilidade de que a ausência de associação não foi decorrente de um erro de classificação não diferencial. Cabe salientar que as médias de lipídios plasmáticos foram semelhantes a resultados encontrados em outros estudos na América Latina 17,18.

Em relação às perdas de acompanhamento, não acreditamos que a impossibilidade de 
acompanhar todos os membros da coorte original tenha enviesado os resultados, a menos que a associação entre as exposições estudadas e o perfil lipídico seja diferente nos não encontrados. Perdas de acompanhamento, mesmo que a distribuição tenha variado de acordo com o estado da doença ou características do baseline, não enviesariam a estimativa de risco. O viés ocorreria somente se as categorias da doença variassem de acordo com as características do baseline.

Ao contrário do sugerido pela teoria da programação fetal, o baixo IMC materno pré-gestacional - um fator claramente associado com a restrição intra-uterina 15 - esteve associado com menores níveis de colesterol total, LDL e nãoHDL. No entanto, esta associação foi mediada pela dieta, composição corporal do adolescente e o nível sócio-econômico. Após controle para IMC, conteúdo de gordura na dieta e anos de escolaridade do adolescente, a diferença observada nos níveis de colesterol não-HDL entre os filhos de mães com IMC no quartil inferior e aqueles cujo IMC materno estava no quartil superior passou de 2,4 para $0,6 \mathrm{mg} / \mathrm{dL}$, e o valor do $\mathrm{p}$ de heterogeneidade mudou de 0,02 para 0,23. Ressalta-se que resultados similares foram observados para colesterol total e LDL.

Mi et al. 19, na China, avaliaram indivíduos nascidos entre 1948 e 1954, período no qual grande parte da população chinesa estava cronicamente subnutrida como conseqüência do fim da Segunda Guerra Mundial e da subseqüente Guerra Civil que ocorreu naquele país. O IMC materno no primeiro trimestre de gestação foi inversamente associado com os níveis de colesterol total e LDL aos 45 anos de idade 19. Já Forrester et al. 20, na Jamaica, relataram que o colesterol total em indivíduos com idades entre 6 e 16 anos estava positivamente associado com o IMC materno no primeiro trimestre da gestação. Finalmente, Kuzawa et al. 21, nas Filipinas, observaram que medidas gestacionais maternas da área de gordura no braço mesmo após ajuste para antropometria do adolescente e dieta estavam relacionadas: (a) negativamente com o colesterol LDL e positivamente com o HDL no sexo masculino; e (b) positivamente com o colesterol total e o LDL no sexo feminino.

O tabagismo materno na gestação é considerado como um dos fatores de risco modificáveis mais importantes para a determinação do crescimento intra-uterino 16 , estando associado com uma redução de 150 a $250 \mathrm{~g}$ no peso ao nascer 10 . Jaddoe e colaboradores verificaram que, na infância, a colesterolemia era maior nos filhos de mães não fumantes, enquanto na adolescência não foi observada associação. Na idade adulta, por sua vez, o colesterol total foi maior nos filhos de mães fumantes 22 . No nosso estudo, mesmo analisando os dados de acordo com a freqüência e a intensidade de fumo materno, não foi possível encontrar uma associação.

$O$ retardo de crescimento intra-uterino se caracteriza por uma redução no crescimento fetal em razão de uma agressão ocorrida no útero 16 . Neste estudo, não houve associação entre nascer pequeno para a idade gestacional, um indicador de retardo de crescimento intra-uterino, e o perfil lipídico na adolescência, estando os resultados consistentes com outros estudos realizados em países de renda média e alta 23,24,25,26.

As evidências da literatura sugerem que o crescimento intra-uterino não está associado com o metabolismo lipídico em crianças e adolescentes. Por outro lado, o resultado de Jaddoe et al. 22 sugere que o efeito do crescimento intrauterino sobre os lipídios plasmáticos pode ser modificado pela idade do indivíduo, similarmente ao constatado com a amamentação 27. Alguns estudos sugerem que o metabolismo do colesterol é modificado à medida que a idade aumenta 28,29. Nos humanos, o colesterol LDL plasmático aumenta aproximadamente $40 \%$ dos 20 aos 60 anos de idade 30 . Igualmente, Lane et al. 7, em modelos animais, não encontraram diferenças no metabolismo hepático dos ácidos graxos entre ratos jovens submetidos à insuficiência uteroplacentária e ratos-controles. Entretanto, tais diferenças foram evidenciadas em ratos adultos 7 . Os mecanismos fisiológicos para a mudança não estão totalmente elucidados. Sabe-se que os níveis de LDL plasmáticos são determinados por um balanço entre sua síntese e eliminação 30,31. Tanto em humanos quanto em ratos a remoção plasmática das partículas de LDL é reduzida com o envelhecimento 28,29,32, provavelmente como conseqüência da redução dos receptores hepáticos de LDL (LDLR), acompanhada pela diminuição na decomposição do colesterol junto a ácidos biliares 28,32,33.

Os resultados do nosso estudo, em conjunto com as evidências da literatura, sugerem que o retardo de crescimento intra-uterino não está associado com o metabolismo lipídico na adolescência. Todavia, tendo em vista alguns achados de que esta associação possa ser modificada pela idade, é importante que novas análises sejam replicadas em populações adultas. Os nossos resultados sugerem que o efeito em longo prazo do IMC materno era mediado pela associação dele com o IMC do adolescente, demonstrando a importância de se prevenir o ganho acelerado de peso na infância e adolescência, que estão associados à adiposidade 34 . 


\section{Resumo}

Avaliou-se o efeito do retardo de crescimento intra-uterino e de fatores de risco para o retardo de crescimento intra-uterino sobre o perfil lipídico em adolescentes pertencentes ao estudo de coorte de nascimentos de 1982 de Pelotas, Rio Grande do Sul, Brasil. Em 2000, os participantes do sexo masculino foram identificados no alistamento militar; $79 \%(n=2.250)$ foram entrevistados e 2.089 doaram amostra de sangue. No presente estudo, as variáveis dependentes foram o colesterol total e suas frações (VLDL, LDL, HDL), colesterol não$H D L$, razão $L D L / H D L$ e triglicerídeos. As exposições estudadas foram o retardo de crescimento intra-uterino, o indice de massa corporal (IMC) materno prégestacional e o tabagismo materno durante a gravidez. Após ajuste para fatores de confusão, o colesterol total, $L D L$ e não-HDL foram maiores entre os adolescentes cujo IMC materno pré-gestacional estava no terceiro e quarto quartil. No entanto, tais associações desapareceram após controle para dieta, escolaridade e IMC do adolescente. O retardo de crescimento intra-uterino e o tabagismo materno na gravidez não foram associados com o perfil lipídico aos 18 anos de idade.

Retardo do Crescimento Fetal; Colesterol; Adolescemte

\section{Referências}

1. Jamison DT, Breman JG, Measham AR, Alleyne G, Claeson M, Evans DB, et al. Disease control priorities in developing countries. New York: Oxford University Press; 2006.

2. Third Report of the National Cholesterol Education Program (NCEP) expert panel on detection, evaluation, and treatment of high blood cholesterol in adults (adult treatment panel III) final report. Circulation 2002; 106:3143-421.

3. Barker DJ, Martyn CN, Osmond C, Hales CN, Fall $\mathrm{CH}$. Growth in utero and serum cholesterol concentrations in adult life. BMJ 1993; 307:1524-7.

4. Lawlor DA, Owen CG, Davies AA, Whincup PH, Ebrahim S, Cook DG, et al. Sex differences in the association between birth weight and total cholesterol. A meta-analysis. Ann Epidemiol 2006; 16: 19-25.

5. Victora CG, Horta BL, Post P, Lima RC, De Leon Elizalde JW, Gerson BM, et al. Breast feeding and blood lipid concentrations in male Brazilian adolescents. J Epidemiol Community Health 2006; 60:621-5.

6. Lucas A. Programming by early nutrition: an experimental approach. J Nutr 1998; 128(2 Suppl): 401S-6S.

\section{Colaboradores}

M. C. Restrepo foi responsável pela análise de dados e redação do manuscrito. B. L. Horta e D. P. Gigante participaram da redação e revisão do artigo.

\section{Agradecimentos}

Este estudo foi realizado com recursos da Wellcome Trust (Reino Unido). As fases iniciais do estudo foram financiadas pelo Programa de Apoio a Núcleos de Excelência (PRONEX) do Conselho Nacional de Desenvolvimento Científico e Tecnológico (CNPq), Ministério da Saúde do Brasil, International Development Research Centre (Canadá) e do United Nations Development Fund for Women (Reino Unido).
7. Lane RH, Kelley DE, Gruetzmacher EM, Devaskar SU. Uteroplacental insufficiency alters hepatic fatty acid-metabolizing enzymes in juvenile and adult rats. Am J Physiol Regul Integr Comp Physiol 2001; 280:R183-90.

8. Ozanne SE. Metabolic programming in animals. $\mathrm{Br}$ Med Bull 2001; 60:143-52.

9. Choi GY, Tosh DN, Garg A, Mansano R, Ross MG, Desai M. Gender-specific programmed hepatic lipid dysregulation in intrauterine growth-restricted offspring. Am J Obstet Gynecol 2007; 196: 477e1-7.

10. Kramer MS. Determinants of low birth weight: methodological assessment and meta-analysis. Bull World Health Organ 1987; 65:663-737.

11. Victora CG, Barros FC, Lima RC, Behague DP, Gonçalves H, Horta BL, et al. The Pelotas birth cohort study, Rio Grande do Sul, Brazil, 1982-2001. Cad Saúde Pública 2003; 19:1241-56.

12. Victora CG, Barros FC. Cohort profile: the 1982 Pelotas (Brazil) birth cohort study. Int J Epidemiol 2006; 35:237-42.

13. Friedewald WT, Levy RI, Fredrickson DS. Estimation of concentration of low-density lipoprotein cholesterol in plasma, without use of preparative ultracentrifuge. Clin Chem 1972; 18:499-502. 
14. Williams RL, Creasy RK, Cunningham GC, Hawes WE, Norris FD, Tashiro M. Fetal growth and perinatal viability in California. Obstet Gynecol 1982; 59:624-32.

15. World Health Organization. Physical status: the use and interpretation of anthropometry: report of a WHO expert committee. Geneva: World Health Organization; 1995.

16. Ergaz Z, Avgil M, Ornoy A. Intrauterine growth restriction-etiology and consequences: what do we know about the human situation and experimental animal models? Reprod Toxicol 2005; 20 : 301-22.

17. Moura EC, Castro CM, Mellin AS, Figueiredo DB. Perfil lipídico em escolares de Campinas, SP, Brasil. Rev Saúde Pública 2000; 34:499-505.

18. Irwig MS, Siles X, Gotto JAM, Rifai N, Campos H. Plasma lipids and other cardiovascular risk factors in Costa Rican adolescents. Rev Panam Salud Pública 2000; 8:234-41.

19. Mi J, Law C, Zhang KL, Osmond C, Stein C, Barker D. Effects of infant birthweight and maternal body mass index in pregnancy on components of the insulin resistance syndrome in China. Ann Intern Med 2000; 132:253-60.

20. Forrester TE, Wilks RJ, Bennett FI, Simeon D, Osmond C, Allen M, et al. Fetal growth and cardiovascular risk factors in Jamaican schoolchildren. BMJ 1996; 312:156-60.

21. Kuzawa CW, Adair LS. Lipid profiles in adolescent Filipinos: relation to birth weight and maternal energy status during pregnancy. Am J Clin Nutr 2003; 77:960-6.

22. Jaddoe VW, Ridder MA, van den Elzen AP, Hofman A, Uiterwaal CS, Witteman JC. Maternal smoking in pregnancy is associated with cholesterol development in the offspring: a 27 -years follow-up study. Atherosclerosis 2008; 196:42-8.

23. Decsi T, Erhardt E, Markus A, Burus I, Molnar D. Plasma lipids, phospholipid fatty acids and indices of glycaemia in 10-year-old children born as small-for-gestational-age or preterm infants. Acta Paediatr 1999; 88:500-4.

24. Levitt NS, Lambert EV, Woods D, Hales CN, Andrew R, Seckl JR. Impaired glucose tolerance and elevated blood pressure in low birth weight, nonobese, young south african adults: early programming of cortisol axis. J Clin Endocrinol Metab 2000; 85:4611-8.
25. Evagelidou EN, Giapros VI, Challa AS, Kiortsis DN, Tsatsoulis AA, Andronikou SK. Serum adiponectin levels, insulin resistance, and lipid profile in children born small for gestational age are affected by the severity of growth retardation at birth. Eur J Endocrinol 2007; 156:271-7.

26. Tenhola S, Martikainen A, Rahiala E, Herrgard E, Halonen P, Voutilainen R. Serum lipid concentrations and growth characteristics in 12-year-old children born small for gestational age. Pediatr Res 2000; 48:623-8.

27. Horta BL, Bahl R, Martines JC, Victora CG. Evidence on the long-term effects of breastfeeding. Geneva: World Health Organization; 2007.

28. Ericsson S, Berglund L, Frostegard J, Einarsson K, Angelin B. The influence of age on low density lipoprotein metabolism: effects of cholestyramine treatment in young and old healthy male subjects. J Intern Med 1997; 242:329-37.

29. Galman C, Matasconi M, Persson L, Parini P, Angelin B, Rudling M. Age-induced hypercholesterolemia in the rat relates to reduced elimination but not increased intestinal absorption of cholesterol. Am J Physiol Endocrinol Metab 2007; 293:E737-42.

30. Wilhelmsen L, Johansson S, Rosengren A, Wallin I, Dotevall A, Lappas G. Risk factors for cardiovascular disease during the period 1985-1995 in Göteborg, Sweden. The GOT-MONICA Project. J Intern Med 1997; 242:199-211.

31. Miettinen TA, Gylling H, Vanhanen H, Ollus A. Cholesterol absorption, elimination, and synthesis related to LDL kinetics during varying fat intake in men with different apoprotein E phenotypes. Arterioscler Thromb 1992; 12:1044-52.

32. Field PA, Gibbons GF. Decreased hepatic expression of the low-density lipoprotein (LDL) receptor and LDL receptor-related protein in aging rats is associated with delayed clearance of chylomicrons from the circulation. Metabolism 2000; 49:492-8.

33. Wang DQ. Aging per se is an independent risk factor for cholesterol gallstone formation in gallstone susceptible mice. J Lipid Res 2002; 43:1950-9.

34. Reilly JJ, Methven E, McDowell ZC, Hacking B, Alexander D, Stewart L, et al. Health consequences of obesity. Arch Dis Child 2003; 88:748-52.

Recebido em 19/Nov/2008

Versão reapresentada em 22/Jul/2009

Aprovado em 27/Jul/2009 